\title{
Discretization error-free estimate of low temperature statistical dissociation rates in gas phase: Applications to Lennard-Jones clusters $X_{13-n} Y_{n}(n=0-3)$
}

\author{
Massimo Mella ${ }^{\text {a) }}$ \\ School of Chemistry, Cardiff University, Main Building, Park Place, Cardiff CF10 3AT, United Kingdom
}

(Received 18 January 2008; accepted 9 May 2008; published online 27 June 2008)

In this work, an improved approach for computing cluster dissociation rates using Monte Carlo (MC) simulations is proposed and a discussion is provided on its applicability as a function of environmental variables (e.g., temperature). With an analytical transformation of the integrals required to compute variational transition state theory (vTST) dissociation rates, MC estimates of the expectation value for the Dirac delta $\delta\left(q_{r c}-q_{c}\right)$ have been made free of the discretization error that is present when a prelimit form for $\delta$ is used. As a by-product of this transformation, the statistical error associated with $\left\langle\delta\left(q_{r c}-q_{c}\right)\right\rangle$ is reduced making this step in the calculation of vTST rates substantially more efficient (by a factor of $4-2500$, roughly). The improved MC procedure is subsequently employed to compute the dissociation rate for Lennard-Jones clusters $X_{13-n} Y_{n}$ $(n=0-3)$ as a function of temperature $(T)$, composition, and $X-Y$ interaction strength. The $X_{13-n} Y_{n}$ family has been previously studied as prototypical set of systems for which it may be possible to select and stabilize structures different from the icosahedral global minimum of $X_{13}$. It was found that both the dissociation rate and the dissociation mechanism, as suggested by the statistical simulations, present a marked dependence on $n, T$, and the nature of $Y$. In particular, it was found that a vacancy is preferentially formed close to a surface impurity when the $X-Y$ interaction is weaker than the $X-X$ one whatever the temperature. Differently, the mechanism was found to depend on $T$ for stronger $X-Y$ interactions, with vacancies being formed opposite to surface impurities at higher temperature. These behaviors are a reflex of the important role played by the surface fluctuations in defining the properties of clusters. () 2008 American Institute of Physics. [DOI: $10.1063 / 1.2937914]$

\section{INTRODUCTION}

Dissociation of atomic and molecular clusters plays a pivotal role in the description of several important phenomena such as the nucleation of liquid droplets or metal aggregates from supersaturated vapor phases. Indeed, knowing the relative concentration of clusters of different sizes and the value of their dissociation rate constant $k^{d}$ makes it possible to compute their condensation or growth rate. With this in mind, it is therefore clear why a substantial theoretical effort has been focused on the development of sophisticated theories or efficient computational methods capable of accurately predicting $k^{d}$ as a function of environmental variables.

One such method is the approach developed by Schenter et al., ${ }^{1}$ who proposed the use of variational transition state theory ${ }^{2}$ (vTST) to compute the rate constant $k_{m}^{d}(T)$ for the dissociation process

$$
M_{m} \rightarrow M_{m-1}+M
$$

in the NTV ensemble. With the assumption of a spherical separatrix of radius $r$ centered on the cluster center of mass, it was possible to show that $k_{m}^{d}(T)$ is proportional to $\partial F / \partial r, F$ being the free energy of a cluster enclosed inside the separatrix. As a consequence of this result, $k_{m}^{d}(T)$ is proved to be

\footnotetext{
${ }^{a)}$ Electronic mail: mellam@cardiff.ac.uk.
}

proportional to the pressure exerted by the cluster on the containing sphere, a fact that paves the way for the use of classical Monte Carlo (MC) simulations in estimating $k_{m}^{d}(T)$. Besides, the variational nature of vTST is exploited not only to minimize $\partial F / \partial r$ (hence the rate constant) but also to provide an estimate of the cluster radius.

A more direct approach to the calculation of $k_{m}^{d}(T)$ starts from the fundamental TST equation for a monomolecular process $^{3,4}$

$$
k(T)=\frac{\int d \mathbf{x} d \mathbf{p} \delta[f(S)] H_{>}(\dot{S}) \dot{S} e^{-\beta \mathcal{H}}}{\int d \mathbf{x} d \mathbf{p} H[f(S)] e^{-\beta \mathcal{H}}},
$$

where $H$ is the Heaviside function, $\mathcal{H}$ is the Hamilton function for the system, $S$ is the reaction coordinate, $f(S)=0$ defines the separatrix, and $\beta=\left(k_{B} T\right)^{-1}$. In Eq. (2), $H_{>}(\dot{S})$ selects the trajectories that, starting on the surface $(\delta[f(S)])$ separating the reactant $(R)$ from the products $(P)$, are leaving the $R$ region, while $H[f(S)]$ selects only points in configuration space within the volume associated with $R$. In the case of a dissociation process, a useful reaction coordinate is provided by the distance between the center of mass of the dissociating moiety and of the remaining cluster $\left(q_{r c}\right)$, so that the dividing surface is defined by the node of $F(S)=q_{r c}-s$, where $s$ is the position along $q_{r c}$ at which the critical surface is located. For this choice of the reaction coordinate and 
assuming that $\mathcal{H}=\mathcal{T}(\mathbf{p})+\mathcal{V}(\mathbf{x})$, it is possible to recast Eq. (2) into the simpler form

$$
k_{m}^{d}(T)=\frac{\left\langle\left|\dot{q}_{r c}\right|\right\rangle_{T}}{2} \frac{\int_{R} d \mathbf{x} \delta\left(q_{r c}-s\right) e^{-\beta \mathcal{V}(\mathbf{x})}}{\int_{R} d \mathbf{x} e^{-\beta \mathcal{V}(\mathbf{x})}} .
$$

Here, the phase space average in Eq. (2) is conveniently reduced to a configuration space one that could be evaluated using metropolis MC (MMC) sampling, ${ }^{5}$ and $\left\langle\mid \dot{q}_{r c}\right\rangle_{T}$ is the average value of the time derivatives of the reaction coordinate in the $N V T$ ensemble. The latter can be computed analytically for our choice of $q_{r c}$ obtaining $\left\langle\left|\dot{q}_{r c}\right|\right\rangle_{T}$ $=\left(2 k_{B} T / \pi \mu\right)^{1 / 2}$, with $\mu$ being the reduced mass of the dissociating moiety with respect to the remaining cluster. ${ }^{6}$ In the case of barrierless dissociation reactions such as the one described by Eq. (1), a key step in evaluating the dissociation rate is represented by choosing the location of the separatrix. This task can be accomplished by exploiting the variational nature of TST and choosing the location of the separatrix in order to minimize the dissociation rate [Eq. (2)] or the integral ratio in Eq. (3) with respect to the "size" of the reactant configuration space $R$ in the framework of the canonical ensemble.

Unfortunately, the direct evaluation of the integral over the configuration space using a straightforward MMC simulation is hampered by two problems, especially in the case of low temperature or high dissociation energy cases. First, the extremely local nature of the Dirac delta forces one to use a prelimit form for $\delta\left(q_{r c}-s\right),{ }^{7}$ the latter being often approximated with a normalized rectangular box of width $\Delta x$. Clearly, this introduces a finite discretization error. Second, a low temperature MMC sampling would visit the regions around $q_{r c}-s=0$ rarely, making the statistical evaluation of the expectation value quite inefficient. The latter problem can be ameliorated either by forcing the MMC simulation to sample the critical region more often with a more diffuse sampling distribution, ${ }^{7}$ by using a stratified sampling approach, ${ }^{8}$ or employing the umbrella sampling (US) scheme originally proposed by Torrie and Valleau and adapted by Grimmelmann et al. ${ }^{10}$ to study the detachment of Xe from metal surfaces (see also the work by Chandler ${ }^{11}$ for a detailed discussion on the relevance of the potential of mean force in condensed phase reactions). The latter scheme has been adapted to the microcanonical ensemble with a modification of the sampling distribution ${ }^{12}$ and shown to provide a substantial gain in efficiency in the calculation of $k^{d}(E)$ for the Zundel cation, $\mathrm{H}_{5}^{+} \mathrm{O}_{2}$.

Despite these improvements, the usage of a prelimit form for $\delta\left(q_{r c}-s\right)$ is still far from being ideal, an issue that is not only aesthetic but, more importantly, related to a possible increase in efficiency. Indeed, recent work in the field of electronic structure quantum MC simulations has led to the development of improved estimators for $\delta\left(\mathbf{x}-\mathbf{x}_{0}\right)$ with much lower variance than the prelimit form. ${ }^{13,14}$ Interestingly, the new estimators proposed in both Refs. 13 and 14 use all available samples, even though weighted differently as a function of $\left|\mathbf{x}-\mathbf{x}_{0}\right|$. This is in sharp contrast with the prelimit approach, where, assuming that an US window of width $L$ is used to force the sampling around $q_{r c}-s=0$ and that $\delta\left(q_{r c}-s\right)$ is approximated with a bin of width $\Delta x$, only a fraction $\Delta x / L$ of the samples is used to estimate the integral ratio in Eq. (3).

With the aim of estimating statistical dissociation rates for large clusters, this work presents a discretization errorfree approach for the calculation of $\left\langle\delta\left(q_{r c}-s\right)\right\rangle$ with MMC simulations. As shown in Sec. II, this is based on an analytical transformation of the integrals in Eq. (3) and allows one to use an appropriate variance reduction technique. In Sec. III, the performance of the new estimator is compared to the prelimit form of the Dirac delta using, first, an analytically solvable model composed by two particles and, second, a cluster composed of six Lennard-Jones particles $\left(\mathrm{LJ}_{6}\right)$ with a potential chosen to mimic the two-body $\mathrm{Ne}-\mathrm{Ne}$ interaction. The new method is subsequently employed to estimate the dissociation rate of $X_{13-n} Y_{n}$, a family of LJ clusters, as a function of $T$ and composition. These results are discussed in Sec. IV and Sec. V. Finally, Sec. VI presents our conclusions and suggests future applications for the new estimator in the realm of cluster chemical physics.

\section{THEORY AND METHODS}

Starting from the fundamental equation [Eq. (3)] and the discussion in Ref. 12, in the following we provide the working equations that make transparent the use of US in this context and that allow one to estimate $k^{d}$ free of discretization errors. Let us start defining the boxcar distribution

$$
\omega\left(q_{r c}, r_{i}, s\right)= \begin{cases}1 & \left(r_{i} \leqslant q_{r c} \leqslant s\right) \\ 0 & \left(q_{\mathrm{rc}} \leqslant r_{i}\right),\end{cases}
$$

where $s$ gives the location of the Dirac delta along $q_{r c}$ (i.e., of the reflecting surface that keeps the sampled points inside the $R$ region). Assuming a series of $r_{i}(i=0, n)$ such that $r_{i-1}<r_{i}<s$ and $r_{0}=0$ (i.e., free sampling), one can exploit the extreme locality of the Dirac delta to rewrite Eq. (3) as

$$
\begin{aligned}
k_{m}^{d}(T)= & \frac{\left\langle\left|\dot{q}_{r c}\right|\right\rangle_{T}}{2} \prod_{i=1}^{n} I_{i-1}\left(r_{i}, s\right) \\
& \times \frac{\int_{R} d \mathbf{x} \omega\left(q_{r c}, r_{n}, s\right) \delta\left(q_{r c}-s\right) e^{-\beta \mathcal{V}(\mathbf{x})}}{\int_{R} d \mathbf{x} \omega\left(q_{r c}, r_{n}, s\right) e^{-\beta \mathcal{V}(\mathbf{x})}},
\end{aligned}
$$

where

$$
I_{i-1}\left(r_{i}, s\right)=\frac{\int_{R} d \mathbf{x} \omega\left(q_{r c}, r_{i}, s\right) e^{-\beta \mathcal{V}(\mathbf{x})}}{\int_{R} d \mathbf{x} \omega\left(q_{r c}, r_{i-1}, s\right) e^{-\beta \mathcal{V}(\mathbf{x})}} .
$$

We notice that this way of recasting Eq. (3) requires the calculation of a set of overlap integrals $\left(I_{i-1}\left(r_{i}, s\right)\right)$ between distributions differing only by their range of definition. In each of those, $r_{i-1}$ defines the lowest value of $q_{r c}$ allowed during the MMC sampling. Clearly, appropriately chosen values for $r_{i-1}$ are needed to efficiently estimate the total overlap $\langle S\rangle=\prod_{i=1}^{n} I_{i-1}\left(r_{i}, s\right)$. The latter can also be interpreted as a ratio between partition functions, from which the reversible work needed to separate a molecule from the rest of the cluster by, at least, the distance $r_{n}$ can be computed. At this stage, it is important to mention the conditions under which Eq. (5) and the ones presented in the rest of this section are valid. In this respect, the range of temperature for which Eq. 
(5) can be used must be such that the dissociation of a molecule from the cluster is a rare event (i.e., the probability of finding the dissociating moiety at $s$ is very small) with the remaining $M_{m-1}$ species satisfying at least one of the possible cluster definitions (for a discussion on this issue, see Ref. 15). Another way of recasting this condition is to ask for the geometrical fluctuations of the surface particles to be of size comparable with the ones in the core of the cluster, the different magnitude of such fluctuation being important to rationalize the peculiar thermodynamics behavior of clusters when compared with bulk matter. ${ }^{16}$ The fulfillment of this hypothesis allows one to interpret the results of the MMC simulations on the basis of a compact cluster-molecule fragmentation, therefore avoiding the possibility of a partial or complete cluster evaporation that may lead to a false fulfillment of the dissociation condition. This chance is easily monitored by computing either molecule-center-of-mass distributions or the instantaneous bond order for each molecule. Alternatively, a good indication of the failure of the rare event hypothesis is provided by the disappearance of the minimum of $k_{m}^{d}(T)$ as a function of $s$ and a nonexponential behavior for $\langle S\rangle$ as a function of $T$.

In order to make progress with respect to Eq. (5), let us focus on the remaining integral ratio. With our choice of reaction coordinate, it is possible to substitute Cartesian coordinates with Jacobi ones so that the vector connecting the center of mass of the dissociating moiety and of the remaining cluster appears explicitly as an integration variable. Recalling that the Jacobian of the transformation from Cartesian to Jacobi coordinates is unit, the integral ratio becomes

$$
\begin{aligned}
\left\langle\delta\left(q_{r c}-s\right)\right\rangle_{n}\left(r_{n}, s\right) & =\frac{\int_{R} d \mathbf{x} \omega\left(q_{r c}, r_{n}, s\right) \delta\left(q_{r c}-s\right) e^{-\beta \mathcal{V}(\mathbf{x})}}{\int_{R} d \mathbf{x} \omega\left(q_{r c}, r_{n}, s\right) e^{-\beta \mathcal{V}(\mathbf{x})}} \\
& =\frac{\int_{R} d \mathbf{q}_{r c} d \mathbf{Q} \omega\left(q_{r c}, r_{n}, s\right) \delta\left(q_{r c}-s\right) e^{-\beta \mathcal{V}\left(\mathbf{q}_{r c}, \mathbf{Q}\right)}}{\int_{R} d \mathbf{q}_{r c} d \mathbf{Q} \omega\left(q_{r c}, r_{n}, s\right) e^{-\beta \mathcal{V}\left(\mathbf{q}_{r c}, \mathbf{Q}\right)}},
\end{aligned}
$$

where $\mathbf{q}_{r c}$ is the vector whose magnitude represents the reaction coordinate and $\mathbf{Q}$ the remaining set of Jacobi coordinates. In this form, it is possible to use the integral identity

$$
\int_{V} f(\mathbf{x}) \delta[g(\mathbf{x})] d^{n} \mathbf{x}=\int_{\partial V} \frac{f(\mathbf{x})}{|\nabla g|} d^{n-1} \mathbf{x},
$$

where $\partial V$ is the surface implicitly defined by $g(\mathbf{x})=0$ and $\mathbf{x}$ $\in \mathcal{R}^{n}$. In our case, the surface is a sphere defined by $q_{r c}-s$ $=0$ and $\left|\nabla_{\mathbf{q}_{r c}} g\right|=1$. Thus, the volume element on the right hand side of Eq. (8) becomes $d^{n-1} \mathbf{x}=s^{2} d \Omega d \mathbf{Q}$, where we have introduced the surface element $d \partial V=s^{2} d \Omega$, with $d \Omega$ representing the solid angle element. Inserting Eq. (8) in Eq. (7) and making use of the new form for $d^{n-1} \mathbf{x}$, one gets

$$
\left\langle\delta\left(q_{r c}-s\right)\right\rangle_{n}\left(r_{n}, s\right)=\frac{s^{2} \int_{R} d \Omega d \mathbf{Q} e^{-\beta \mathcal{V}(s, \Omega, \mathbf{Q})}}{\int_{R} d \mathbf{q}_{r c} d \mathbf{Q} \omega\left(q_{r c}, r_{n}, s\right) e^{-\beta \mathcal{V}\left(\mathbf{q}_{r c}, \mathbf{Q}\right)}},
$$

where, at the denominator, $d \mathbf{q}_{r c}=q_{r c}^{2} d q_{r c} d \Omega$.

In order to make Eq. (9) amenable to MMC simulations, one needs to recast it in terms of a standard expectation value calculation, a task that is accomplished by choosing an integrable function $N\left(q_{r c}\right)$ such that $\int_{r_{n}}^{s} N\left(q_{r c}\right) q_{r c}^{2} d q_{r c}=1$ and inserting the latter integral in the numerator. By means of straightforward manipulations, one finally gets

$$
\left\langle\delta\left(q_{r c}-s\right)\right\rangle_{n}\left(r_{n}, s\right)=\frac{s^{2} \int_{R} d \mathbf{q}_{r c} d \mathbf{Q} \omega\left(q_{r c}, r_{n}, s\right) N\left(q_{r c}\right) e^{-\beta\left[\mathcal{V}(s, \Omega, \mathbf{Q})-\mathcal{V}\left(q_{r c}, \Omega, \mathbf{Q}\right)\right]} e^{-\beta \mathcal{V}\left(q_{r c}, \Omega, \mathbf{Q}\right)}}{\int_{R} d \mathbf{q}_{r c} d \mathbf{Q} \omega\left(q_{r c}, r_{n}, s\right) e^{-\beta \mathcal{V}\left(q_{r c}, \Omega, \mathbf{Q}\right)}}
$$

which suggests the canonical average of $\Delta(s, \beta, N)$ $=s^{2} N\left(q_{r c}\right) e^{-\beta\left[\mathcal{V}(s, \Omega, \mathbf{Q})-\mathcal{V}\left(q_{r c}, \Omega, \mathbf{Q}\right)\right]}$ over the volume constrained distribution $\omega\left(q_{r c}, r_{n}, s\right) e^{-\beta \mathcal{V}\left(q_{r c}, \Omega, \mathbf{Q}\right)}$ as new estimator for $\left\langle\delta\left(q_{r c}-s\right)\right\rangle_{n}\left(r_{n}, s\right)$. Here, it is important to stress that the angular coordinates $\Omega$ appearing in $\mathcal{V}(s, \Omega, \mathbf{Q})$ are chosen accordingly to the MMC sampling of the distribution $\mathcal{V}\left(q_{r c}, \Omega, \mathbf{Q}\right)$, i.e., representing $\mathbf{q}_{r c}$ in spherical coordinates and using the angular part to define $\Omega$.

The transformation used to obtain Eq. (10) bears some resemblance with a similar analytical approach proposed by Dumont and Jain. ${ }^{17}$ There are, however, two important differences, namely, the introduction of the weight function $N\left(q_{r c}\right)$ and the fact that the integral over $N\left(q_{r c}\right)$ is over a range of $q_{r c}$ inside the location of the separatrix instead than outside. Whereas the usefulness of introducing $N\left(q_{r c}\right)$ will be discussed in the following, it is mentioned here that the internal integration range allows one to use many more samples than other way. This is expected to improve the statistical error of the $\left\langle\delta\left(q_{r c}-s\right)\right\rangle_{n}\left(r_{n}, s\right)$ estimate.
From Eq. (10), one can easily outline the steps of a possible simulation procedure:

(i) select the temperature $T$ (or $\beta=\left(k_{B} T\right)^{-1}$ );

(ii) select the location $s$ of the critical surface along the reaction coordinate $q_{r c}$ and of $r_{1}$, the lower limit of the first overlapping window [Eqs. (4)-(6)]. $s$ is also used to constrain the MMC sampling by rejecting displacements for which the distance $q_{r c}$ becomes larger than $s$;

(iii) simulate the cluster with atoms constrained to have $q_{r c}<s$ and compute $I_{0}\left(r_{1}, s\right)$ using all atoms to improve statistics (notice, however, that $I_{i-1}$ are all defined with respect to a specific dissociating moiety);

(iv) choose the atom that is "driven out" by the US procedure and compute the set of integrals $I_{i-1}$ for $i=2$ $-n$ with it. Notice that this approach effectively deals only with one of the possible dissociation channels, so that the $k_{m}^{d}(T)$ obtained using this and the following 
steps refers to the dissociation of a "tagged" particle. Assuming ergodic behavior for the MMC simulations, the total dissociation rate for $M_{m}$ is obtained by multiplying the rate obtained with this procedure by the channel degeneracy;

(v) while running the MMC simulation sampling $\omega e^{-\beta \mathcal{V}\left(q_{r c}, \Omega, \mathbf{Q}\right)}$ for the chosen atom, compute $\mathbf{q}_{r c}$ for it and generate the vector $\mathbf{q}_{r c}^{\prime}=s \mathbf{q}_{r c} /\left|\mathbf{q}_{r c}\right|$ placing the dissociating atom on the surface with the correct angular coordinates;

(vi) shift the position of the dissociating atom using $\mathbf{q}_{r c}^{\prime}$ and compute the value of the potential $\mathcal{V}$ in the shifted configuration; and

(vii) average the value of $s^{2} N\left(q_{r c}\right) e^{-\beta\left[\mathcal{V}(s, \Omega, \mathbf{Q})-\mathcal{V}\left(q_{r c}, \Omega, \mathbf{Q}\right)\right]}$ over all samples.

From Eq. (10) and the sequence of steps outlined above, it should be clear that the original source of fluctuation present in the prelimit estimate of the delta has now been substituted by the fluctuation of $\Delta(s, \beta, N)$. However, one also realizes that the new estimator enjoys a zero variance in the limit of $N\left(q_{r c}\right) e^{-\beta\left[\mathcal{V}(s, \Omega, \mathbf{Q})-\mathcal{V}\left(\mathbf{q}_{r c}, \mathbf{Q}\right)\right]} \simeq$ const. The latter cannot be obtained for the prelimit estimator even in the limit of no interactions between the dissociating atom and the rest of the cluster. To clarify this point further, let us assume that for $r_{n} \leqslant q_{r c} \leqslant s$ one has $\beta\left|\mathcal{V}(s, \Omega, \mathbf{Q})-\mathcal{V}\left(\mathbf{q}_{r c}, \mathbf{Q}\right)\right| \simeq 0$ and that $N\left(q_{r c}\right)$ is chosen to be constant over the interval. In this case, the fluctuation of $\Delta(s, \beta, N)$ is largely reduced, whereas the prelimit estimator would still suffer from the limited amount of sampling falling in the thin spherical crown defined by the interval $s-\Delta x \leqslant q_{r c} \leqslant s$.

In cases where $\left.\beta\left|\mathcal{V}(s, \Omega, \mathbf{Q})-\mathcal{V}\left(\mathbf{q}_{r c}, \mathbf{Q}\right)\right|>0\right)$, one can chose the analytical form of $N\left(q_{r c}\right)$ to reduce the variance of the estimator. Many possibilities are available for this goal, the best form for $N\left(q_{r c}\right)$ being likely to depend on the specific cluster details. Nevertheless, it is possible to provide guidelines for the case in which a large value $s$ is chosen, considering the difference $\mathcal{V}(s, \Omega, \mathbf{Q})-\mathcal{V}\left(\mathbf{q}_{r c}, \mathbf{Q}\right)$ to be a function of $q_{r c}$ only. This is equivalent to a mean field approach that neglects the instantaneous structural fluctuation of the cluster. With this assumption, one would be allowed to interpret $e^{-\beta\left[\mathcal{V}(s, \Omega, \mathbf{Q})-\mathcal{V}\left(\mathbf{q}_{r c}, \mathbf{Q}\right)\right]}$ as the ratio between the angular averaged probability density of the dissociating particle at $s$ and $q_{r c}$ and to choose $N\left(q_{r c}\right)$ as proportional to the radial probability density $\rho\left(q_{r c}\right)$ defined by

$$
\rho\left(q_{r c}\right)=\frac{\int_{R} d \Omega d \mathbf{Q} \omega\left(q_{r c}, r_{n}, s\right) e^{-\beta \mathcal{V}\left(\mathbf{q}_{r c}, \mathbf{Q}\right)}}{\int_{R} d \mathbf{q}_{r c} d \mathbf{Q} \omega\left(q_{r c}, r_{n}, s\right) e^{-\beta \mathcal{V}\left(\mathbf{q}_{r c}, \mathbf{Q}\right)}}
$$

Another advantage provided by $N\left(q_{r c}\right)$ relates to the exponential nature of $\Delta(s, \beta, N)$ : a carefully chosen form may produce a more compact distribution of its values, thus reducing possible biases due to the limited set of statistical measurements performed during the simulation. ${ }^{18}$ Indeed, a more compact distribution of weights may help in satisfying the hypothesis of the central limit theorem, the latter being implicitly assumed in computing averages over a set of mean values.

\section{TESTS ON MODEL SYSTEMS}

In this section, simulations on two model systems are used to test the performance of $\Delta(s, \beta, N)$ when compared to the prelimit form of the Dirac delta.

\section{A. Dimer with square-well interaction}

To make sure that the simulation method was correctly implemented, the simple test case provided by two identical particles interacting by means of the square-well potential

$$
\mathcal{V}\left(\mathbf{x}_{1}, \mathbf{x}_{2}\right)= \begin{cases}V_{0} & \left(\left|\mathbf{x}_{1}-\mathbf{x}_{2}\right| \leqslant \xi\right) \\ 0 & \left(\left|\mathbf{x}_{1}-\mathbf{x}_{2}\right|>\xi\right)\end{cases}
$$

has been studied. Here, $V_{0}$ is the well depth, $\xi$ is the distance at which the interaction terminates, and $\mathbf{x}_{i}$ is the absolute position of the $i$ th particle. For this choice of $\mathcal{V}$, the expectation value $\left\langle\delta\left(q_{r c}-s\right)\right\rangle_{n}\left(r_{0}, s\right)$ can be computed analytically, providing us with a useful reference to test the algorithm. Using Eq. (3) and $\mathcal{V}$ as defined in Eq. (12), one gets

$$
\left\langle\delta\left(q_{r c}-s\right)\right\rangle_{n}\left(r_{0}, s\right)=\frac{3 s^{2}}{\left(e^{-\beta V_{0}}-1\right) \xi^{3}+s^{3}},
$$

where $\beta=\left(k_{B} T\right)^{-1}$ and it has been assumed that $s \geqslant \xi$.

The top panel of Fig. 1 presents the average values of $\left\langle\delta\left(q_{r c}-s\right)\right\rangle$ for the specific choice of parameters $V_{0}$ $=-0.047$ hartree and $\xi=3$ bohrs at two different temperatures $\left(k_{B} T=0.005,0.009\right.$ hartree $)$. These results were obtained by straightforward MMC simulations (i.e., no US was employed) using both the prelimit estimator and Eq. (10) with $N\left(q_{r c}\right)=$ const. To reduce the computational cost of the MMC simulations, $\left\langle\delta\left(q_{r c}-s\right)\right\rangle$ values at different $s_{i}$ 's were computed simultaneously constraining the MMC sampling within a sphere of radius $s_{\max }$ (the largest $s_{i}$ chosen) and using only configurations falling within $q_{r c} \leqslant s_{i}$ to estimate the Dirac delta at $s_{i}$. Although this implementation may have an effect on the statistical precision of $\left\langle\delta\left(q_{r c}-s\right)\right\rangle$ in region where $s$ is short, it allows one to effectively compute the integral ratio in Eq. (3) for different sizes of the reactant space in a single simulation. As a consequence, choosing the location of the separatrix by means of the variational criterion could be carried out during a postsimulation processing stage. Standard errors were estimated by means of nine statistical independent simulations, the latter being composed by 100 blocks of $10^{5} \mathrm{MC}$ steps each. The length of attempted displacements was adjusted in order to obtain an acceptance ratio of $35 \%-60 \%$ for the MMC moves. The analytical results provided by Eq. (13) are also shown for comparison.

From this figure, it is clear that the two estimators [i.e., prelimit and $\Delta(s, \beta, N\rangle$ with $N\left(q_{r c}\right\rangle=$ const] are in good agreement with $\left\langle\delta\left(q_{r c}-s\right)\right\rangle$ from Eq. (13), a fact that confirms the robustness of both procedures and their correct implementation. It also appears that Eq. (10) is able to produce fairly more precise results than the prelimit estimator at low temperature, a result that is supported by the absolute value of the relative standard error $\sigma /\langle\delta\rangle$ at $k_{B} T=0.005$ hartree (lower panel of Fig. 1). This is particularly evident at small 

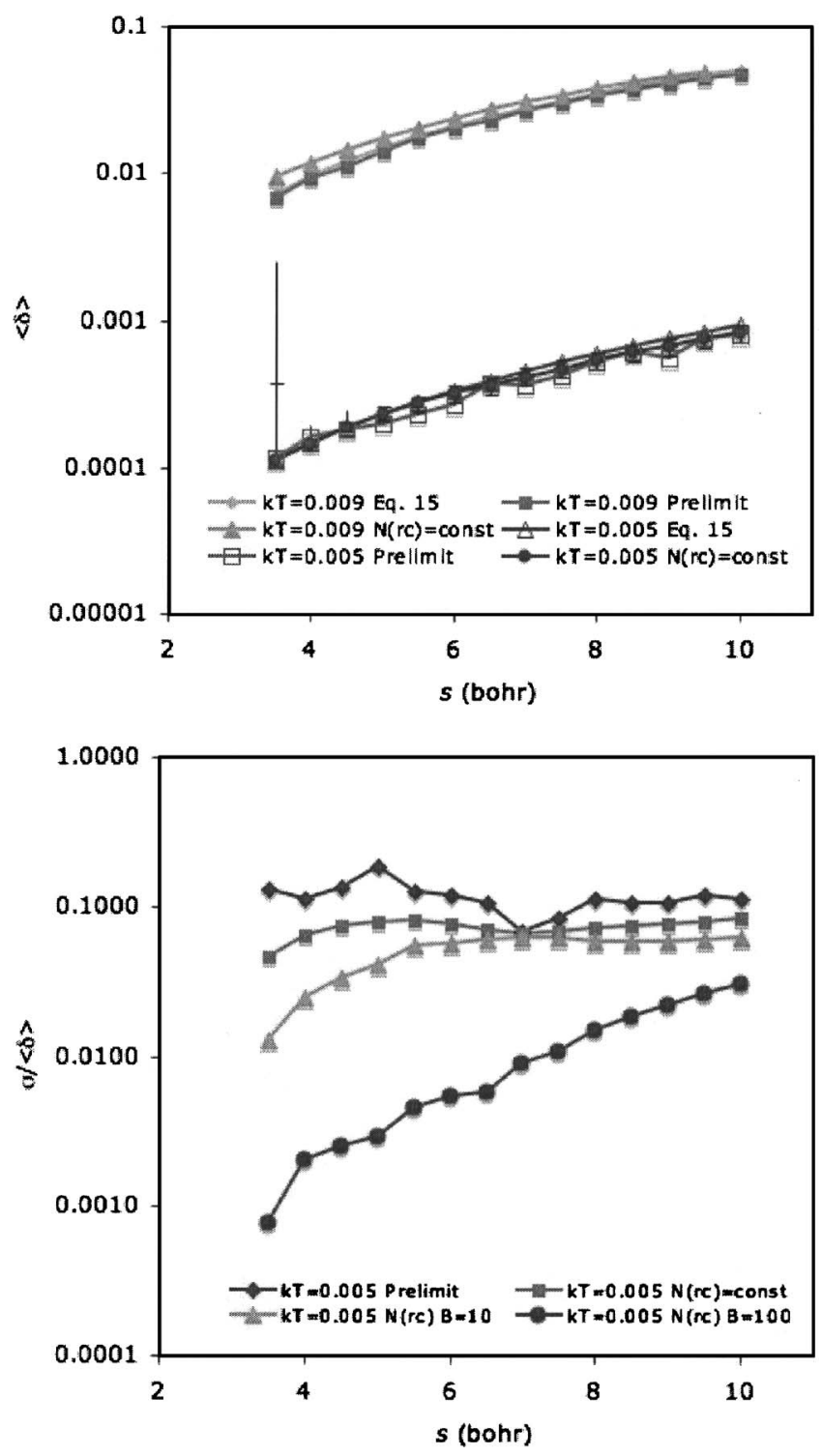

FIG. 1. Top panel: values of $\left\langle\delta\left(q_{r c}-s\right)\right\rangle$ as a function of $s$ for the square-well dimer [Eq. (12)] for $\beta=0.005$ and 0.009 hartree. Lower panel: relative standard error for $\left\langle\delta\left(q_{r c}-s\right)\right\rangle$ for the prelimit estimator and Eq. (10) (see main text) at $k_{B} T=0.005$ hartree. In the case of Eq. (10), three different $B$ values for the weight function $N\left(q_{r c}\right)$ were used [see Eq. (14)].

values of $s$, which are sampled less frequently during the MMC simulation. To provide one with indications on the effect of $N\left(q_{r c}\right)$ on the statistical precision of our estimates, results obtained using the natural weight function

$$
N\left(q_{r c}\right)= \begin{cases}B & \left(0 \leqslant q_{r c} \leqslant \xi\right) \\ 1 & \left(q_{r c}>\xi\right),\end{cases}
$$

with $B=10,100$ are also shown in the lower panel of Fig. 1 . For this choice of $B$, the improvements in efficiency obtained by using Eq. (10) become even more apparent, the standard error being reduced by a factor of 3-10 when $B=10$ and 7-200 when $B=100$. These improvements in precision directly translate into a substantially reduced computational cost (roughly, a factor of 9-100 for $B=10$ and 49-10 000 for $B=100)$, stressing the importance of an appropriate choice for $N\left(q_{r c}\right)$. Notice that the additional computational cost due to the evaluation of $N\left(q_{r c}\right)$ and $\mathcal{V}(s, \Omega, \mathbf{Q})$ during the MMC simulation is made negligible by computing $\Delta(s, \beta, N)$ only after the serial correlation between configurations has vanished.

\section{B. $\mathrm{Ne}_{6}$ dissociation}

To test further the improvements introduced by using $\Delta(s, \beta, N)$ as an estimator for $\left\langle\delta\left(q_{r c}-s\right)\right\rangle$, simulations were carried out on a system composed of six particles interacting by means of a standard LJ potential whose well depth $\left(\epsilon=1.1274 \times 10^{-4}\right.$ hartree $)$ and width $(\sigma=5.194 \mathrm{bohr})$ were chosen to mimic the $\mathrm{Ne}-\mathrm{Ne}$ interaction. For this case, the straightforward MMC sampling as done in the square-well dimer is hindered by the long range intermolecular forces between the particles and by the low temperature range we would like to explore $(3-10 \mathrm{~K})$, the latter spanning a range of phases going from solidlike to fluidlike. Thus, the stepwise US scheme provided by Eq. (5) should be used to "drive" the dissociating particle far from the cluster and to compute $\left\langle\delta\left(q_{r c}-s\right)\right\rangle$ over the appropriate range of $s$. Since the performance of the US scheme in driving the sampling toward the dissociation region and in estimating the overlap integrals [Eq. (6)] has already been discussed in literature, ${ }^{10,12}$ in this section we focus only on comparing the performance of the different estimators over the $r_{n} \leqslant q_{r c}$ $\leqslant s_{\max }$ window (in this case $r_{n}=9.5$ and $s_{\max }=20$ bohrs). To cover the relevant range of $T$ and to help alleviate problems with broken ergodicity, simultaneous sampling at several temperatures was conducted using a standard implementation of the parallel tempering (PT) scheme. ${ }^{19}$ We would expect the calculation of $\left\langle\delta\left(q_{r c}-s\right)\right\rangle$ to benefit from PT simulations when simulating systems whose energy landscape is characterized by a multifunnel character or that may undergo phase changes over the relevant temperature range. With a procedure similar to the one used in the square-well interaction case, the value of $\left\langle\delta\left(q_{r c}-s\right)\right\rangle$ at several locations $\left(s_{i}\right)$ was computed for all temperatures during the same simulation.

Another issue present for this system, which is present for more general molecular clusters as well, is the selection of the analytical form for the weight function $N\left(q_{r c}\right)$. Provided that the locations $s_{i}$ of the generalized TS's are far enough from the center of mass of the remaining cluster, a suitable form for $N\left(q_{r c}\right)$ could be built fitting the shape of $\rho$ as obtained by short preliminary simulations. To do so, we employed a piecewise exponential form (i.e., the logarithm of $\rho$ was piecewise fitted with straight lines). With this choice, the normalization of $N\left(q_{r c}\right)$ can be easily computed using standard integral formulas.

The top panel of Fig. 2 presents the results for $\left\langle\delta\left(q_{r c}-s\right)\right\rangle$ over $r_{n} \leqslant q_{r c} \leqslant s_{\max }$ using the prelimit form of the Dirac delta and Eq. (10). In the latter case, both $N\left(q_{r c}\right)$ $=$ const or the piecewise representation just discussed were used. Simulations were run using a protocol similar to the square-well case and standard errors were obtained using seven statistically independent simulations. As found previously, the three estimates agree well at all temperatures, indicating the correct implementation of the procedure. At low temperature, it is apparent that the prelimit form presents a 

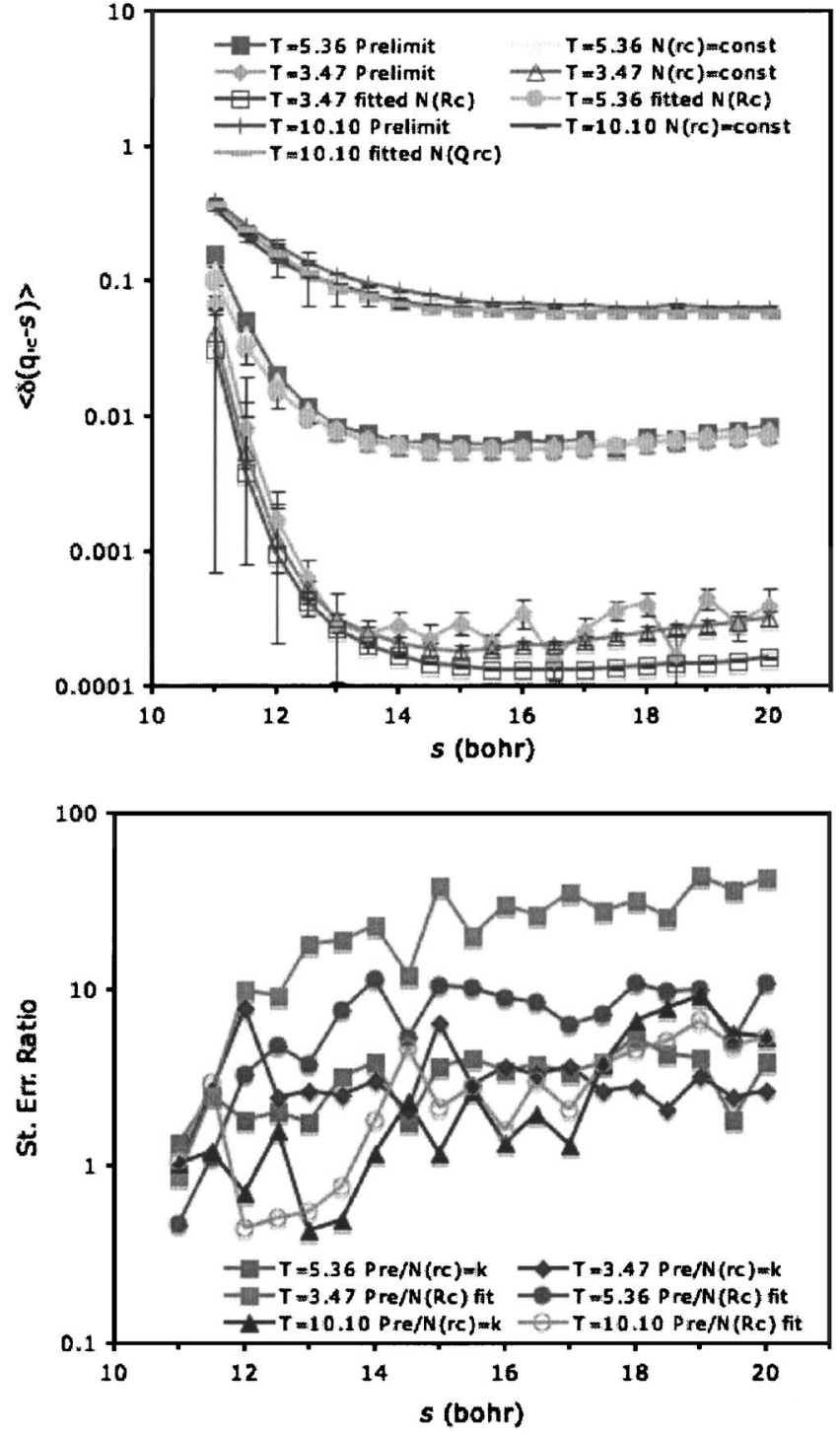

FIG. 2. Top panel: $\left\langle\delta\left(q_{r c}-s\right)\right\rangle$ for the Ne-like $\mathrm{LJ}_{6}$ at three different temperatures (in kelvins). $N\left(q_{r c}\right)=$ const indicates the use of a constant weight function, whereas fitted $N\left(q_{r c}\right)$ indicates the use of a piecewise approximation for $N\left(q_{r c}\right)$. Lower panel: ratio of the standard errors obtained computing $\left\langle\delta\left(q_{r c}-s\right)\right\rangle$ with Eq. (10) and the prelimit estimator. Temperatures in kelvins.

much noisier behavior and a substantially larger standard error. This impression is validated by the lower panel of Fig. 2, where the ratio between standard errors is shown. Worth mentioning at this stage is the fact that over all range of temperature explored during the simulations with $q_{r c} \geqslant r_{n}$ no evidence was found of multiple particle dissociation and that $\mathrm{Ne}_{5}$ remained quite compact as evidenced by plotting cluster center-of-mass-particle distribution functions. This is, of course, a consequence of the large ratio between the evaporation energy (equivalent to roughly $128 \mathrm{~K}$ ) and the simulation temperature. Additionally, the top panel of Fig. 2 shows the presence of a minimum for $\left\langle\delta\left(q_{r c}-s\right)\right\rangle$ for all three simulations, a good indication of the validity of the TST hypothesis as discussed in the Introduction.

Overall, the results shown in the lower panel of Fig. 2 suggest that both choices of $N\left(q_{r c}\right)$ provide one with a substantial improvement with respect to the prelimit estimator, a fact that is clearly evident at $T=3.47 \mathrm{~K}$ and $T=5.36 \mathrm{~K}$. In these cases, the increased precision provided by $\Delta(s, \beta, N)$ translates into a large reduction in the number of samples (roughly, by a factor of 9-1600) needed to obtain a chosen statistical accuracy in the range of $s$ around the minimum of $\left\langle\delta\left(q_{r c}-s\right)\right\rangle$ where the sampling is scarce. For systems whose interaction potential is written as a sum of pairwise potentials, this improvement should open up the chance of simulating clusters 3-40 times larger than the ones amenable to the prelimit form. Needless to say, this factor may be further increased with a more sensible choice of $N\left(q_{r c}\right)$.

\section{DISSOCIATION OF COMPACT LJ-LIKE $X_{13-n} Y_{n}$ CLUSTERS}

To demonstrate the capability of the proposed estimator to deal efficiently with larger systems, the dissociation rate $k^{d}(T)$ of the 13 particle LJ cluster $X_{13-n} Y_{n}$ (Ref. 20) has been computed. The topology of the $X_{13-n} Y_{n}$ potential energy surface was shown to differ substantially from the $X_{13}$ one, these differences being dependent on both $n$ and the strength of the $Y-Y$ and $X-Y$ interactions. ${ }^{20}$ In fact, both the geometry of the global minima and the height of its isomerization barriers could be tuned with an appropriate selection of those parameters. Isomerization rates were also computed using a harmonic version of TST (Ref. 21) to provide one with information on the lifetime $\tau$ of the clusters. The latter is an important element in the design of cluster motives.

The possibility of cluster thermal dissociation is another element that should be kept in consideration, even though it may be slower than isomerization due to higher energy barriers. However, dissociation (to $X_{12-n} Y_{n}+X$ ) and isomerization barriers appear to have comparable heights for $X_{13-n} Y_{n}{ }^{22}$ therefore suggesting the dissociation as a possible competing channel. Besides, investigating the behavior of $k^{d}(T)$ for $X_{13-n} Y_{n}$ as a function of the nature of $Y, n$, and $T$ may contribute to a better understanding of the role played by each variable in defining the dissociation rate. ${ }^{23}$

For this family of systems, the LJ interaction between particles is characterized by different energies and length scales (respectively, $\epsilon_{X X}$ and $\sigma_{X X}$ for $X-X$, and $\epsilon_{Y Y}$ and $\sigma_{Y Y}$ for $Y-Y)$, with the $X-Y$ interaction parameters being chosen using the standard mixing rules $\epsilon_{X Y}=\left(\epsilon_{X X} \epsilon_{Y Y}\right)^{1 / 2}$ and $\sigma_{X Y}$ $=\left(\sigma_{X X}+\sigma_{Y Y}\right) / 2 . \quad \epsilon_{X X}$ and $\sigma_{X X}\left(3.7935 \times 10^{-4}\right.$ hartree and 6.4354 bohrs, respectively) were chosen to represent the $\mathrm{Ar}-\mathrm{Ar}$ interaction, with the remaining parameters being defined on the basis of the ratios $(\sigma, \epsilon)=\left(\sigma_{Y Y} / \sigma_{X X}, \epsilon_{Y Y} / \epsilon_{X X}\right)$. As done in Ref. 20, we used four different $(\sigma, \epsilon)$ pairs $[(0.8,0.5),(0.8,1.0),(0.8,1.5)$, and $(0.8,2.0)]$ and $n=1-3$ to explore the effect on $k^{d}(T)$ in a systematic way. The structure of the lowest energy isomers obtained by quenching low temperature PT samples for all $(\sigma, \epsilon)$ pairs and $n=1-3$ is shown in Fig. 3 together with $X_{13}$; the lowest energy isomers conserve the icosahedral structure featured by $X_{13}$ in all cases.

PT-MMC simulations, covering the range of temperature $5-25 \mathrm{~K}$, were run as described earlier for $\mathrm{Ne}_{6}$. In the chosen range of temperature, the phase of all clusters changes from solidlike to fluidlike as in the $\mathrm{Ne}_{6}$ case. The US was carried out with $r_{i}$ 's $(i=1-63)$ in the range $7.7-14$ bohrs $\left(r_{i}-r_{i-1}\right.$ 

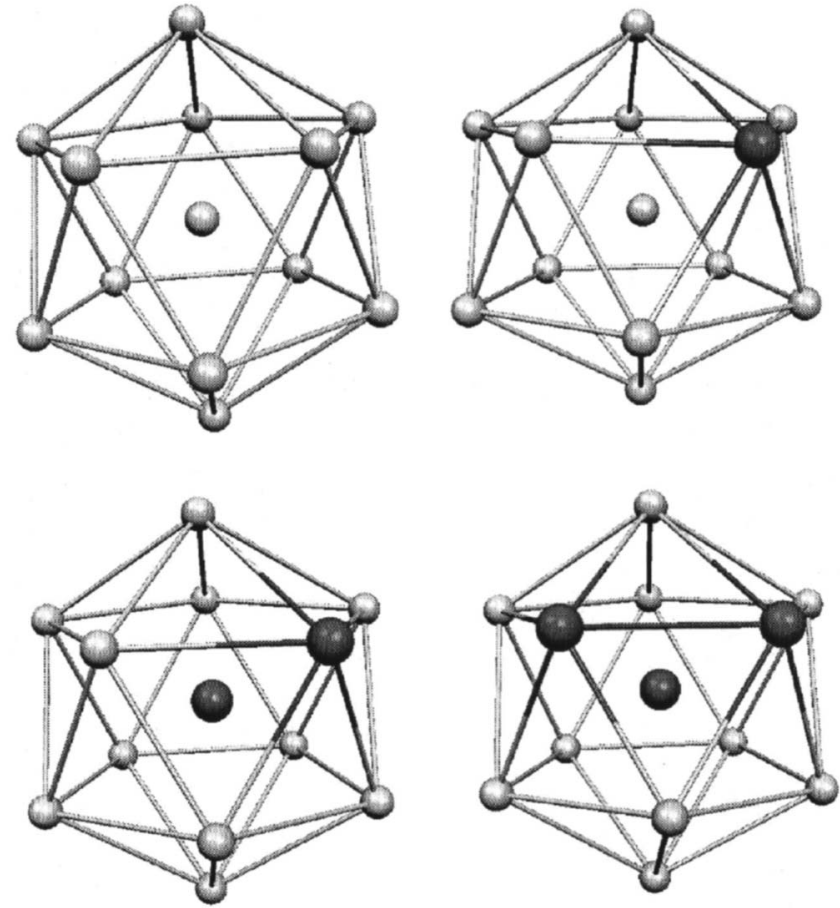

FIG. 3. Lowest energy isomers of $X_{13-n} Y_{n}$ as obtained by local minimization of low temperature samples during PT runs. These differ from the global minimum isomers only for $n=1$, in which case the $Y$ impurity resides in the center of the cluster. Notice that the lowest energy isomer is independent of $(\sigma, \epsilon)$ and depends only on the composition parameter $n$. Also shown is the global minimum of $\mathrm{LJ}_{13}\left(X_{13}\right.$ in our notation).

$=0.1 \mathrm{bohr}$ ), a set of parameters that was roughly optimized to obtain the highest possible efficiency from the MC simulations. The standard error for $\langle S\rangle$ was estimated using the error propagation formula $\sigma^{2}(T)=[\langle S\rangle]^{2} \sum_{i}\left(\sigma_{i-1}^{2}(T)\right) / I_{i-1}^{2}$. Here, $\sigma_{i-1}(T)$ is the standard error associated with $I_{i-1}$, the value of the $i$ th ratio of integrals [see Eq. (6).$^{24}$ The standard error for $\left\langle\delta\left(q_{r c}-s\right)\right\rangle$, computed at several values of $s$, was estimated using a set of seven independent simulations carried out over the window $r_{n}=14, s_{\max }=40$ bohrs. Worth mentioning is the fact that with this choice of simulation parameters and window width, we were not able to collect sufficient statistics to obtain a prelimit estimate for $\left\langle\delta\left(q_{r c}-s\right)\right\rangle$ in the appropriate range of $s$. In our view, this indicates, once again, the need for the new estimator to extend the range of applicability of Eq. (3) and the fact that our simulations are indeed correctly sampling the appropriate configuration space (i.e., the one in which the two dissociated fragments are represented by a single particle and a compact $M_{m-1}$ cluster). As before, further evidences for the correctness of our sampling were found by looking at the behavior of $\left\langle\delta\left(q_{r c}-s\right)\right\rangle$ versus $s$ and at the cluster distribution functions. To provide an estimate for the computational time involved in the calculations, we point out that collecting $10^{7}$ PT-MMC samples for 20 temperatures required roughly 13 min on a single processor Intel Xeon $2.0 \mathrm{GHz}$, so that the total cost was roughly $15 \mathrm{~h}$ per species.

Figures 4 and 5 show the average lifetime $\tau$ (in seconds) for the four families of $X_{13-n} Y_{n}$. Since we focus primarily on the dissociation of an $X$ atom from the cluster, the lifetime was estimated as $\tau=\left[(13-n) k^{d}(T)\right]^{-1}$, where $k^{d}(T)$ has units of $\mathrm{s}^{-1}$. In all cases, $\tau$ was computed using the lowest value of $k_{n}^{d}(T)$ [or of the integral ratio in Eq. (3)] as a function of $s$ over an equispaced set $s_{i}\left(\Delta s_{i, i-1}=1\right.$ bohrs $)$ of separatrix locations

We start discussing the results by noticing that $X_{13-n} Y_{n}$ species with $(\sigma, \epsilon)=(0.8,0.5)$ and $(0.8,1.0)$ [panels (a) and (b), Fig. 4] have shorter $\tau$ than $X_{13}$ for all $n$, whereas $(\sigma, \epsilon)$ $=(0.8,1.5)$ and $(0.8,2.0)$ species [panels (a) and (b), Fig. 5] have a longer lifetime. The substantially shorter $\tau$ and its decrease upon increasing $n$ for $(\sigma, \epsilon)=(0.8,0.5)$ can be understood by referring to the cluster thermal dissociation energy $\left(\Delta V^{d}(T)\right)$, estimated as the difference between the average potential energy of a free species and of an aggregate with $q_{r c} \geqslant 14$ bohrs. $\Delta V^{d}(T)$ substantially decreases upon increasing $n$, indicating the effect of the reduced $X-Y$ well depth as a possible root cause of this finding [panel (a), Fig. 4] and justifying the fact that low temperature configurations for $X_{13-n} Y_{n}$ present the vacancy left by the dissociating $X$ atom close to the $Y$ impurities. ${ }^{25}$ Upon increasing the temperature, this feature is gradually reduced; vacancies also appear further away from the $Y$ atoms, a fact that is due to the increased probability of visiting higher energy configurations during the MMC sampling.

The reversed trend of $\tau(T)$ versus $n$ for $(\sigma, \epsilon)$ $=(0.8,1.0)$ is also in agreement with the $\Delta V^{d}(T)$ values, which indicate the $X$ atoms to be slightly more bound when $n=2$ and 3 . At the atomic level, this may be interpreted as due to a substantial release of frustration in $X_{12} Y$ thanks to a structural relaxation upon dissociation. Supporting this interpretation, it is the fact that a vacancy was found to form mainly opposite to the surface impurities when $n=1$ and close to them when $n=2$ and 3 .

As for $(\sigma, \epsilon)=(0.8,1.5)$ and $(0.8,2.0)$, the increased strength of the $X-Y$ interaction and the slightly higher $\Delta V^{d}(T)$ values for $X_{13-n} Y_{n}$ than for $X_{13}$ fully account for the longer lifetime and for the fact that the vacancy if often created opposite to the surface $Y$ atoms. This mechanism clearly allows the system to minimize the energetic cost associated with the dissociation process. In the less frequent cases in which the dissociating $X$ atom leaves a vacancy near a surface $Y$ impurity, the cluster was found to orient so as to maximize the $X-Y$ interaction, a finding suggesting the possibility that nonstatistical effects may play a role in defining the value of $k^{d}(T)$ for these two families of systems. It would therefore be conceivable to improve the choice of a spherical separatrix, allowing it to deform in response to the cluster structure.

Comparing the results in Figs. 4 and 5 with the data shown in Fig. 14 of Ref. 20 for the isomerization lifetime, one notices similar trends for these two quantities as a function of $n$ and $(\sigma, \epsilon)$, with both $\tau$ 's increasing upon increasing the interaction strength and $n$. Differences in lifetime of up to 20 orders of magnitude between isomerization (faster) and dissociation are, however, present. At the moment, it is found difficult to reconcile these differences in the light of the similar barrier heights and of the two different TST approaches used to estimate the lifetimes. 

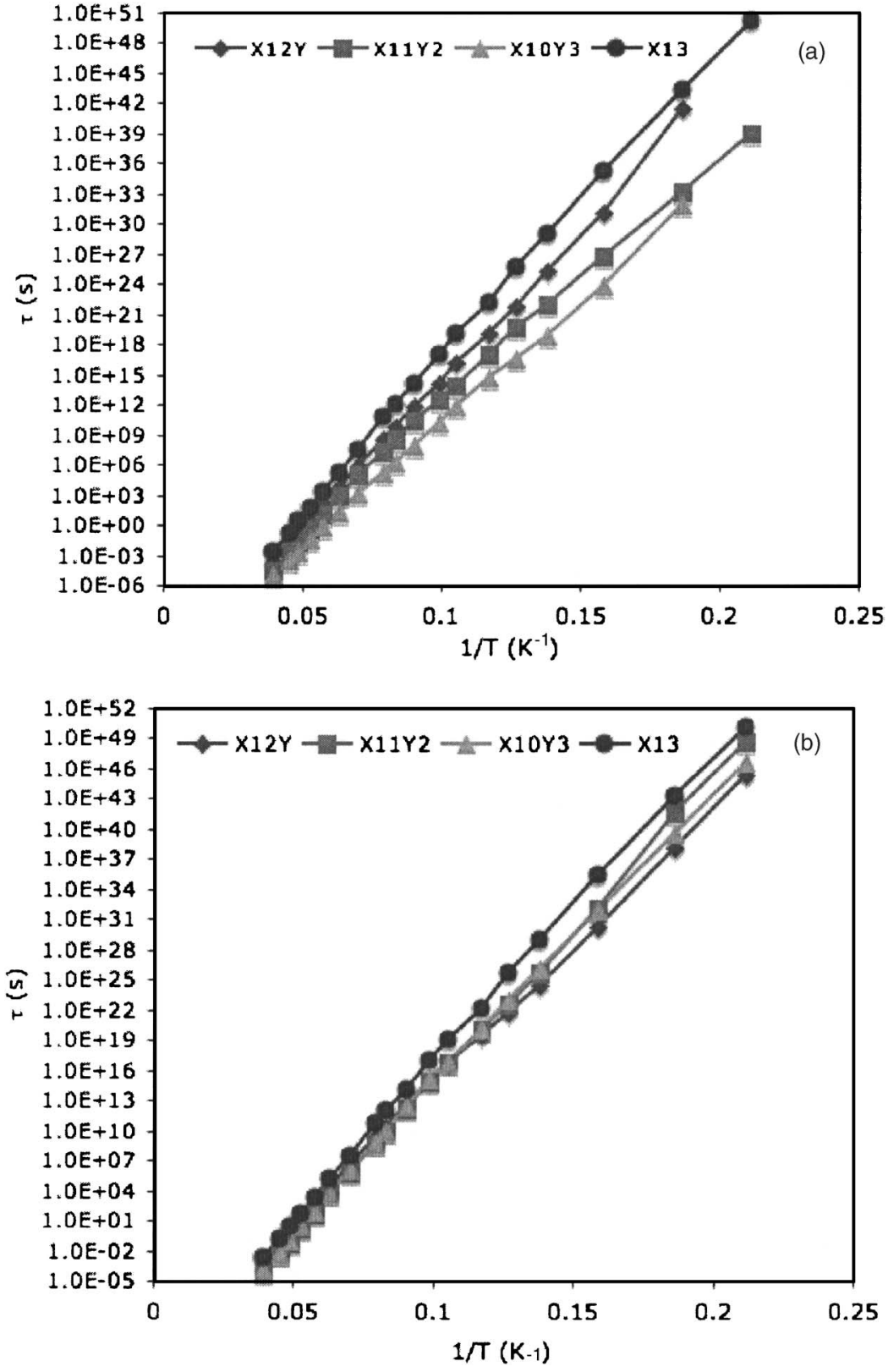

FIG. 4. $X_{13-n} Y_{n}$ lifetime $\tau$ for the dissociation process $X_{13-n} Y_{n} \rightarrow X_{12-n} Y_{n}+X$ as a function of the temperature $T$, the number $n$ of impurities, and the strength of the $Y-Y$ interaction as defined in the main text. (a) $(\sigma, \epsilon)$ $=(0.8,0.5)$ and $(\mathrm{b})(\sigma, \epsilon)=(0.8,1.0)$.

\section{DISSOCIATION OF LJ-LIKE $X_{13-n} Y_{n}$ CLUSTERS WITH EXOTIC STRUCTURES}

As shown in Fig. 3, the clusters so far investigated featured compact structure as low energy isomers, clearly a good choice in terms of structural stability. Clusters with low coordinated surface atoms may, instead, be more active in processes such as heterogeneous catalysis despite the possibly lower lifetimes than their compact counterparts. To investigate quantitatively the possible correlation between structure and dissociation lifetime in the framework of the same model system, ${ }^{20}(\sigma, \epsilon)=(0.45,0.65)$ for $X_{12} Y,(\sigma, \epsilon)$ $=(0.65,1.0)$ for $X_{11} Y_{2}$, and $(\sigma, \epsilon)=(0.42,1.0)$ for $X_{10} Y_{3}$ were chosen as suitable parameter sets. The corresponding global minima are shown in Fig. 6.

The simulation protocol and temperature range used for the species in Fig. 6 are identical to the one employed in the previous section. Also in these cases, the cluster phase changes from solidlike to fluidlike over the spanned tempera- ture range. Noteworthy, $X_{12} Y$ undergoes a solid-solid phase change (a peak in the $C_{v}$ is found around $3-8 \mathrm{~K}$ ), converting the structure shown in Fig. 6 into the structure shown in Fig. 3 (top right). The latter is the global minimum when $\sigma$ $<0.35,{ }^{20}$ a fact that suggests that these two species have similar free energies despite the different structures.

Figure 7 shows both the overlap $\langle S\rangle$ and the final value of the lifetime as a function of $1 / T$. $\langle S\rangle$ appears to be fairly small and closely follows an exponential behavior as a function of $1 / T$ in spite of the less compact cluster structures, a finding supporting the suitability of TST in computing the $X_{13-n} Y_{n}$ dissociation rates. Besides, no indication for multiple dissociations was found during the simulations. Quantitatively, $\langle S\rangle$ for $X_{13-n} Y_{n}(n=1-3)$ is several orders of magnitude larger than for the "pure" $X_{13}$, already suggesting a larger $k^{d}$ for the doped species. The relative value of $\Delta V^{d}(T)(T=5 \mathrm{~K})$ for the four species also supports this idea; $\Delta V^{d}(T)$ is $0.001838(1), 0.000956(1), 0.001386(4)$, and 

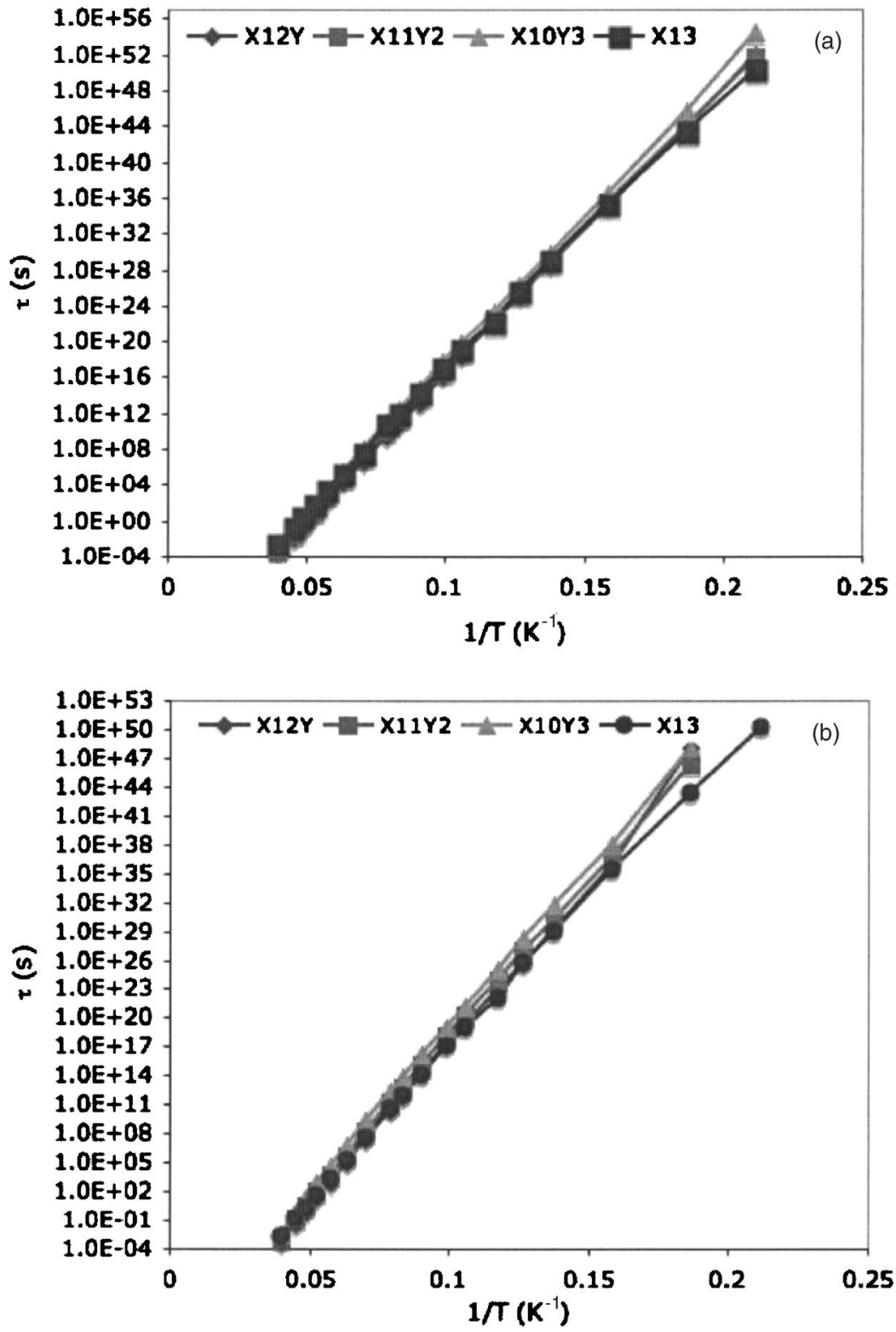

FIG. 5. $X_{13-n} Y_{n}$ lifetime $\tau$ for the dissociation process $X_{13-n} Y_{n} \rightarrow X_{12-n} Y_{n}+X$ as a function of the temperature $T$, the number $n$ of impurities, and the strength of the $Y-Y$ interaction as defined in the main text. (a) $(\sigma, \epsilon)$ $=(0.8,1.5)$ and $(\mathrm{b})(\sigma, \epsilon)=(0.8,2.0)$.
$0.000856(5)$ hartree for $X_{13}, X_{12} Y, X_{11} Y_{2}$, and $X_{10} Y_{3}$, respectively, values that follow a trend similar to the $\langle S\rangle(1 / T)$ one in agreement with simple statistical mechanics arguments.

The logarithm of the average lifetimes $\ln (\tau)$ (in $s$, lower panel of Fig. 7) follows closely the linear trend set by $\Delta V^{d}(T)$ and $\ln (\langle S\rangle)$, the only exception being the low temperature $(5-8 \mathrm{~K})$ rate of $X_{12} Y$. In this region, one notices a change in slope of $\log [\tau(1 / T)]$ over the solid-solid transformation temperature range, the decrease in the slope of $\log [\tau(1 / T)]$ upon decreasing $1 / T$ being justified by a transformation into a more compact structure for $X_{12} Y$. A similar effect, although less evident from the graph, is also seen for $X_{11} Y_{2}$ in the range $0.08 \leqslant 1 / T \leqslant 0.12 \mathrm{~K}^{-1}$, with an accompanying structural modification of the cluster in the same temperature range.

\section{DISCUSSION AND CONCLUSIONS}

Assuming a spherical separatrix between reactants $\left(M_{m}\right)$ and products $\left(M_{m-1}\right.$ plus $\left.M\right)$, a novel estimator for $\left\langle\delta\left(q_{r c}-s\right)\right\rangle$, an expectation value required to estimate canonical TST cluster dissociation rates, has been derived and implemented for stochastic simulations. This estimator, based on an analytical transformation of the original integral ratio, allows one to eliminate completely the discretization error associated with the usually employed prelimit form for the Dirac delta and, with a sensible choice for the weight function $N\left(q_{r c}\right)$, benefits from a large reduction in the associated variance substantially decreasing the computational costs. For instance, the computational cost of estimating $\left\langle\delta\left(q_{r c}-s\right)\right\rangle$ for $\mathrm{Ne}_{6}$ was reduced by a factor of 20-1600 depending on the system temperature. With the current implementation, the variational nature of TST can be exploited to find the "best" separatrix location by means of a simple postprocessing of the $\left\langle\delta\left(q_{r c}-s_{i}\right)\right\rangle$ data. In our view, this result paves the way for similar calculations on systems 4-40 times larger than previously accessible. Besides, the same approach could easily be extended to compute microcanonical reaction rates with the minor modification suggested in Ref. 12. 

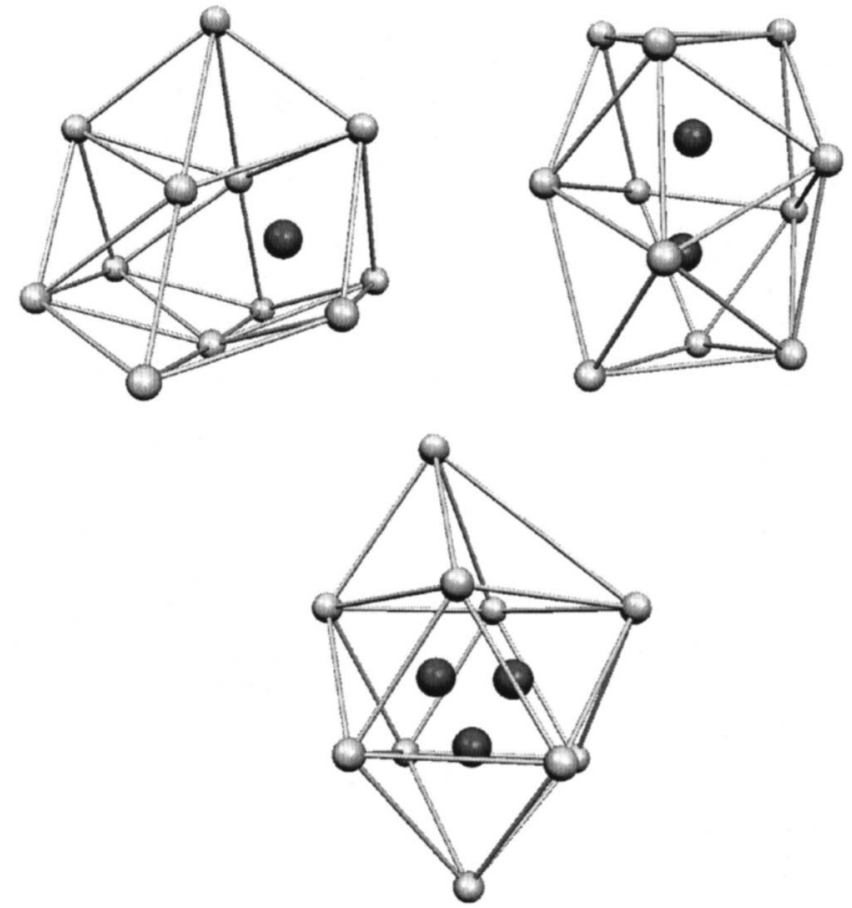

FIG. 6. Lowest energy isomers for $X_{13-n} Y_{n}$ as obtained by local minimization of low temperature samples during PT runs. Interaction parameters are $(0.45,0.65)$ for $X_{12} Y,(0.45,1.0)$ for $X_{11} Y_{2}$, and $(0.42,1.0)$ for $X_{10} Y_{3}$.

The new estimator was subsequently employed to estimate TST dissociation rates for the family of systems $X_{13-n} Y_{n}$ in the temperature range $5 \leqslant T \leqslant 25 \mathrm{~K}$. The behavior of $k^{d}$ versus $T, n$, and interaction strength was discussed using the thermally averaged dissociation energy as guideline. Importantly, the statistical accuracy provided by the new estimator allowed us to highlight subtle changes in the behavior of $\tau$ versus $T$ for $X_{12} Y$ with $(\sigma, \epsilon)=(0.45,0.65)$ (Figs. 6 and 7). These changes were interpreted as due to the onset of solid-solid morphological modification for $X_{13-n} Y_{n}$.

At this stage, it is, however, important to mention that an alternative, and perhaps more complete, explanation for the behaviors of $\tau$ versus $T$ discussed in the previous sections would be available. This rests on the interpretation of $\langle S\rangle$ as a ratio of partition functions, which gives the reversible work needed to pull a molecule apart from the remaining cluster. The latter quantity is minimum due to the reversible conditions (absence of dissipation). In this respect, the weaker surface interaction due to the presence of the impurities allows larger structural fluctuations of the surface particles, therefore increasing the overlap with the dissociating region $\left(q_{r c}>r_{n}\right)$ and reducing the amount of work necessary for the dissociation process. This explanation appears in line with all the findings discussed above, bearing in mind that at higher $T$ the system shows a higher likelihood of performing greater amount of work (molecules dissociating far from weakly interacting impurities). For the case of strongly interacting impurities, wider surface fluctuations are expected to be located opposite to the surface impurities, therefore minimizing the amount of work necessary for the dissociation.

In our view, the successful application of the new estimator to compute TST dissociation rates for 13 atom clusters
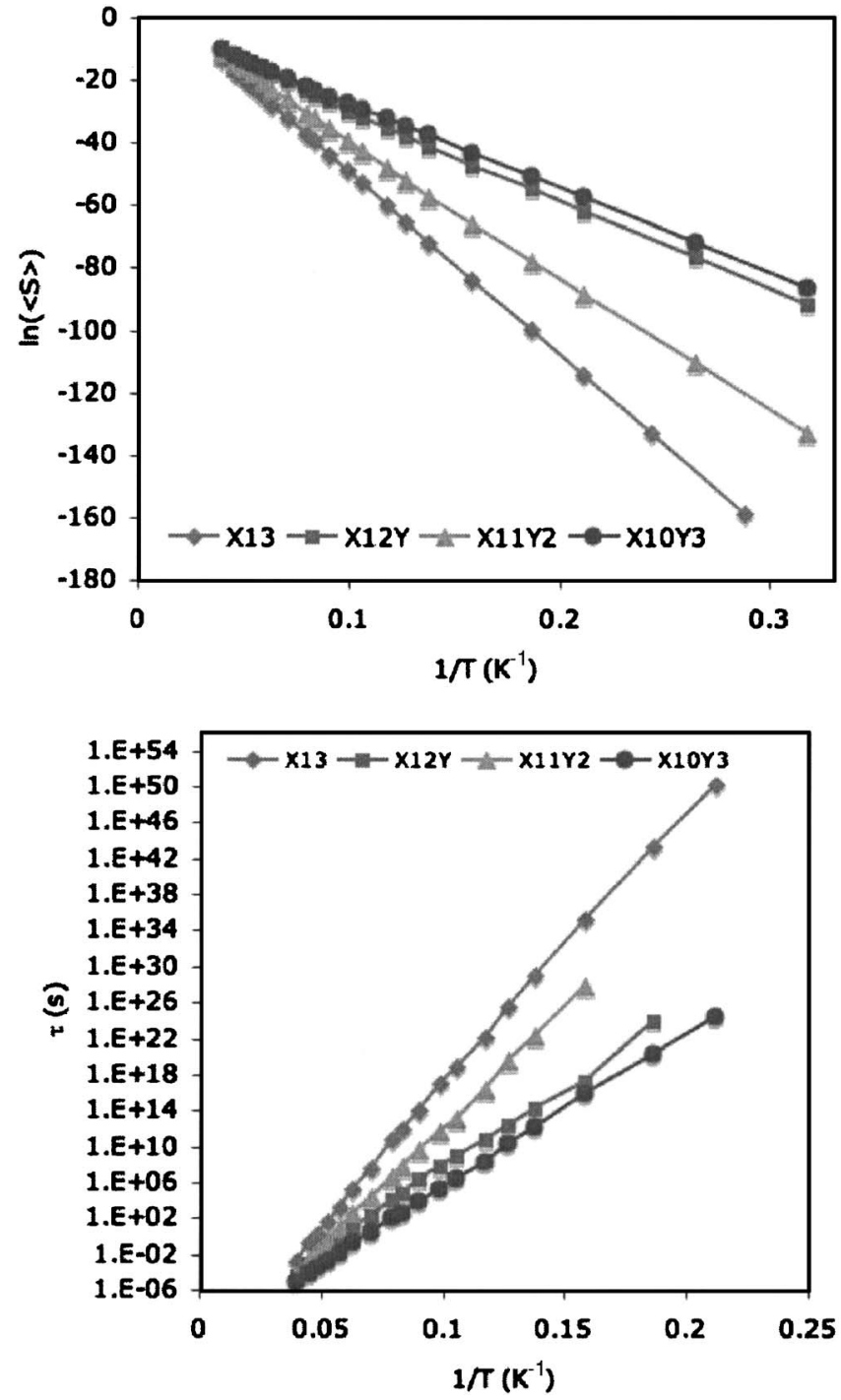

FIG. 7. Top panel: $\langle S\rangle=\prod_{i=1}^{n} I_{i-1}\left(r_{i}, s\right)$ for $X_{13-n} Y_{n}$ as a function of $1 / T$. Lower panel: Lifetime for $X_{13}, X_{12} Y_{2}[(0.45,0.65)], X_{11} Y_{2}[(0.65,1.0)]$, and $X_{10} Y_{3}[(0.42,1.0)]$ as a function of $1 / T$. Temperatures in kelvins.

is a clear indication of the improved performance provided by the combination of a partial analytical integration and MMC sampling. This paves the way for similar calculations on much larger atomic and molecular clusters, the extension of the current implementation to deal with rigid body interaction models being trivial. Indeed, it would be sufficient to define the Jacobi coordinates employed in this work using the Cartesian coordinates of the molecular centers of mass, letting the MMC sampling to carry out the integration over the orientations. In the case of flexible models, instead, one could define the center of mass for $M_{m-1}$ and the dissociating molecule separately and use the simple coordinate transformation $\quad \mathbf{X}_{\text {tot }}=\left(m_{M_{m-1}} \mathbf{x}_{M_{m-1}}+m_{M} \mathbf{x}_{M}\right) /\left(m_{M_{m-1}}+m_{M}\right)$ and $\mathbf{q}_{r c}$ $=-\mathbf{x}_{M_{m-1}}+\mathbf{x}_{M}$ to define the reaction coordinate. Here, $\mathbf{x}_{M_{n-1}}$ and $\mathbf{x}_{M}$ are, respectively, the centers of mass of $M_{m-1}$ and the of dissociating molecule, $m_{M_{m-1}}$ and $m_{M}$ being their masses.

Despite the substantial improvement introduced by the new estimator, two important issues still remain, namely, the cost of the US calculations and the possibility of morphing the shape of the separatrix into a nonspherical one. As for the 
first, it is worth mentioning that we were forced to use 63 US simulations of $10^{7}$ samples each to reach a $10 \%$ precision on $\langle S\rangle$, whereas only seven identical simulations were necessary to reach a similar result for $\left\langle\delta\left(q_{r c}-s\right)\right\rangle$. This fact clearly highlights the need for a more efficient procedure to compute $\langle S\rangle$ [or the ratio of partition functions in Eq. (3)]. Possible approaches to this task are the compensating potential method, ${ }^{10}$ metadynamics, ${ }^{26}$ and the "fast growth" method ${ }^{27}$ associated with Jarzynski's equality. ${ }^{28}$ Whether any one of these represent a more efficient way to compute $\langle S\rangle$ than US is an issue currently under investigation in our laboratory.

In contrast to the previous discussion with respect to US, the issue of the shape of the separatrix concerns more the absolute accuracy of the dissociation rates than the total computational cost. Indeed, our simulations have highlighted a possible change of mechanism, with the location of a vacancy generated by the dissociating atom being a function of T. A similar behavior is expected to be likely for clusters with elongated shapes, suggesting that an ellipsoidal separatrix may be better suited for the task. Thus, further extensions of the approach proposed in this work may be needed to make it more accurate and to avoid the necessity of testing the accuracy of TST rates with molecular dynamics simulations. $^{29}$ Needless to say, larger clusters may have a more spherical shape, automatically ameliorating the performance of a spherical separatrix. This is exactly the case for atomic (e.g., $\left.\mathrm{Au}_{50-100}\right)$ and molecular [e.g., $\left.\left(\mathrm{NH}_{3}\right)_{50-100}\right]$ clusters approaching nanometer size, the calculation of their $k^{d}(T)$ being currently carried out in our laboratory. Needless to say, the thrust in this direction is provided by the technological and scientific importance of both $\mathrm{Au}$ and $\mathrm{NH}_{3}$ clusters.

\section{ACKNOWLEDGMENTS}

The author would like to thank Samuel R. Stone for a careful reading of the manuscript and Gabriele Morosi for useful comments and suggestions. The author acknowledges support from an EPSRC Advanced Research Fellowship (GR/R77803/01).
${ }^{1}$ G. K. Schenter, S. M. Kathmann, and B. C. Garrett, J. Chem. Phys. 110, 7951 (1999).

${ }^{2}$ J. C. Keck, J. Chem. Phys. 32, 1035 (1960).

${ }^{3}$ E. Wigner, Trans. Faraday Soc. 34, 34 (1938).

${ }^{4}$ W. H. Miller, J. Chem. Phys. 61, 1823 (1974).

${ }^{5}$ N. Metropolis, A. W. Rosembluth, M. N. Rosembluth, A. H. Teller, and E. Teller, J. Chem. Phys. 21, 1087 (1953).

${ }^{6}$ H. W. Schranz, L. M. Raff, and D. L. Thompson, Chem. Phys. Lett. 171, 68 (1990).

${ }^{7}$ H. W. Schranz, L. M. Raff, and D. L. Thompson, J. Chem. Phys. 94, 4219 (1991)

${ }^{8}$ D. V. Shalashilin and D. L. Thompson, J. Phys. Chem. A 101, 961 (1997).

${ }^{9}$ G. M. Torrie and J. P. Valleau, J. Comput. Phys. 23, 187 (1977).

${ }^{10}$ E. K. Grimmelmann, J. C. Tully, and E. Helfand, J. Chem. Phys. 74, $5300(1981)$

${ }^{11}$ D. Chandler, J. Chem. Phys. 68, 2959 (1978).

${ }^{12}$ M. Mella, J. Chem. Phys. 124, 104302 (2006).

${ }^{13}$ S. Chiesa, M. Mella, and G. Morosi, Phys. Rev. A 69, 022701 (2004).

${ }^{14}$ J. Toulouse, R. Assaraf, and C. J. Umrigar, J. Chem. Phys. 126, 244112 (2007).

${ }^{15}$ J. Wedekind and D. Reguera, J. Chem. Phys. 127, 154516 (2007).

${ }^{16}$ A. Proykova and R. S. Berry, J. Phys. B 39, R167 (2006).

${ }^{17}$ R. S. Dumont and S. Jain, J. Chem. Phys. 103, 6151 (1995).

${ }^{18}$ M. R. Shirts and V. S. Pande, J. Chem. Phys. 122, 144107 (2005).

${ }^{19}$ R. H. Swendsen and J.-S. Wang, Phys. Rev. Lett. 57, 2607 (1986).

${ }^{20}$ D. Sabo, J. D. Doll, and D. L. Freeman, J. Chem. Phys. 121, 847 (2004).

${ }^{21}$ J. P. Rose and R. S. Berry, J. Chem. Phys. 96, 517 (1992).

${ }^{22}$ This quantity can be roughly estimated by counting the number of nearest neighbors of a surface $X$ atom and comparing it with the isomerization barriers provided by the connectivity diagrams in Ref. 20 .

${ }^{23}$ P. Parneix and P. Bréchignac, J. Chem. Phys. 118, 8234 (2003).

${ }^{24}$ Alternatively, $\sigma^{2}(T)$ could be estimated by directly simulating the dispersion of its values under the assumption of a Gaussian distribution for all $\sigma_{i}(T)$. Tests made on preliminary simulations showed these two procedures to be largely equivalent, and it was therefore decided to present only values of $\sigma^{2}(T)$ obtained with the error propagation formula.

${ }^{25}$ This tendency is stronger in $X_{11} Y_{2}$ and $X_{10} Y_{3}$ than in $X_{12} Y_{1}$ due to the fact that impurities sit in proximity of each other and indicates that even in the presence of a small amount of thermal excitation the dissociation minimum energy path is closely followed.

${ }^{26}$ A. Laio and M. Parrinello, Proc. Natl. Acad. Sci. U.S.A. 99, 12562 (2002).

${ }^{27}$ D. A. Hendrix and C. Jarzynski, J. Chem. Phys. 114, 5974 (2001).

${ }^{28}$ C. Jarzynski, Phys. Rev. Lett. 78, 2690 (1997).

${ }^{29}$ G. K. Schenter, S. M. Kathmann, and B. C. Garrett, J. Chem. Phys. 116, 4275 (2002). 